\title{
Adesão aos protocolos de atendimento para a não infecção de sítio cirúrgico de coluna* $^{*}$
}

\section{Adherence to care protocols for non-infection of the surgical site of the spine}

Cumplimiento de los protocolos de atención para la no infección del sitio quirúrgico de la columna vertebral

\section{Eliane Carlosso Krummenauer ${ }^{\mathrm{I}}$, Jane Dagmar Pollo Renner ${ }^{\mathrm{II}}$, Rochele Mosmann Menezes ${ }^{\mathrm{III}}$, Telmo Tiburcio Fortes Lima ${ }^{\mathrm{IV}}$, Marcelo Carneirov}

\begin{abstract}
Resumo: Objetivo: avaliar a adesão aos protocolos de atendimento para a não infecção de sítio cirúrgico (NISC) de coluna e os fatores associados. Método: estudo transversal realizado por meio da revisão de 60 prontuários de pacientes submetidos à cirurgia de coluna de 2015 a 2019, seu desfecho de não infecção e as condições relacionadas. O estudo foi executado no primeiro semestre de 2020. Resultados: algumas variáveis relacionadas para NISC foram: profilaxia antimicrobiana de 30 a 60 min antes da cirurgia $(R R=0,97 ; p=0,026)$, normotermia $(R R=0,80 ; p=0,050)$, internação pós cirúrgica em Unidade de Terapia Intensiva (até 3 dias) ( $R R=2,00 ; p=0,040)$. A frequência de NISC foi de 83,3\% (50/60) p=0,728). Conclusão: apenas a normotermia foi fator associado a não infecção para NISC na regressão linear. Ressalta-se que a adesão aos processos de trabalho é primordial para a proteção das infecções, reduzir eventos adversos e garantir a segurança.
\end{abstract}

Descritores: Controle de Infecções; Coluna Vertebral; Infecção da Ferida Operatória; Medidas de Prevenção; Segurança do Paciente

Abstract: Objective: to evaluate adherence to treatment protocols for non-surgical site infection (NISC) of the spine and associated factors. Method: cross-sectional study conducted through the review of 60 medical records of

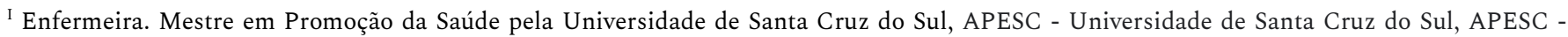
Universidade de Santa Cruz do Sul, RS, Brazil. E-mail: elianek@unisc.br, Orcid: https://orcid.org/0000-0002-8824-4573

II Farmacêutica. Doutora em Biologia Celular e Molecular. Docente dos Departamentos de Ciências da Saúde e Ciências da Vida, Universidade de Santa Cruz do Sul, RS, Brasil. E-mail: janerenner@unisc.br, Orcid: https://orcid.org/0000-0003-0649-7081

III Farmacêutica. Mestre em Promoção da Saúde pela Universidade de Santa Cruz do Sul, RS, Brasil. E-mail: rochelemenezes@unisc.br, Orcid: https://orcid.org/0000-0002-1548-1607

IV Médico. Mestre em Medicina pela Universidade Federal de São Paulo. Neurocirurgião, Hospital Santa Cruz, Santa Cruz do Sul, RS, Brazil. Email: dr.telmotiburcio@yahoo.com.br, Orcid: https://orcid.org/0000-0001-9217-2050

V Médico. Doutor em Medicina pela Universidade Federal do Rio Grande do Sul. Docente dos Departamentos de Ciências da Saúde e Ciências da Vida, Universidade de Santa Cruz do Sul, RS, Brasil. E-mail: marceloc@unisc.br, Orcid: https://orcid.org/0000-0003-3603-1987
}

* Extraído da tese "Avaliação de Campos Adesivos Impregnados com Iodo para Prevenção de Infecções em Cirurgias de Coluna Vertebral”, Programa de Pós-Graduação em Promoção da Saúde, Universidade de Santa Cruz do Sul, UNISC, 2021. 
Adesão aos protocolos de atendimento para a não infecção de sítio cirúrgico de coluna | 2

patients undergoing spinal surgery from 2015 to 2019, its non-infection outcome and related conditions. The study was carried out in the first half of 2020. Results: some variables related to NISC were: antimicrobial prophylaxis from 30 to $60 \mathrm{~min}$ before surgery ( $R \mathrm{R}=0.97 ; \mathrm{p}=0.026$ ), normothermia ( $\mathrm{R}=0.80 ; \mathrm{p}=0.050$ ), post-surgical hospitalization in the Intensive Care Unit (up to 3 days) ( $R R=2.00 ; p=0.040)$. The frequency of NISC was $83.3 \%(50 / 60)$ $\mathrm{p}=0.728$ ). Conclusion: only normothermia was a factor associated with non-infection for NISC in linear regression. It is noteworthy that adherence to work processes is essential to protect against infections, reduce adverse events and ensure safety.

Descriptors: Infection Control; Spine; Surgical Wound Infection; Prevention Measures; Patient Safety

Resumen: Objetivo: Evaluar la adherencia a los protocolos de tratamiento de la infección del sitio no quirúrgico (NISC) de la columna y los factores asociados. Método: estudio transversal realizado mediante la revisión de 60 historias clínicas de pacientes sometidos a cirugía de columna entre 2015 y 2019, su desenlace no infeccioso y afecciones relacionadas. El estudio se realizó en el primer semestre de 2020. Resultados: Algunas variables relacionadas con NISC fueron: profilaxis antimicrobiana de 30 a 60 min antes de la cirugía $(R R=0,97 ; p=0,026)$, normotermia ( $R R=0,80 ; p=0,050$ ), internación posquirúrgica en la Unidad de Cuidados Intensivos (hasta 3 días) $(R R=2,00 ; p=0,040)$. La frecuencia de NISC fue del 83,3\% (50/60) p = 0,728). Conclusión: solo la normotermia fue un factor asociado a la no infección por NISC en regresión lineal. Es de destacar que la adherencia a los procesos de trabajo es fundamental para proteger contra infecciones, reducir los eventos adversos y garantizar la seguridad.

Descriptores: Control de Infecciones; Columna vertebral; Infección de heridas quirúrgicas; Medidas de prevención; Seguridad del paciente

\section{Introdução}

As infecções relacionadas à assistência à saúde (IRAS) são adquiridas por pacientes durante o atendimento e representam o evento adverso mais frequente no âmbito da segurança do paciente em todo o mundo. ${ }^{1-2}$ As infecções de sítio cirúrgico (ISC) são o tipo de IRAS frequentemente monitorado e comum em países de baixa e média renda. Embora menos incidente nos países de alta renda, continua sendo o segundo tipo de IRAS na Europa e nos Estados Unidos (EUA). ${ }^{1}$ Uma pesquisa de prevalência de infecção descobriu que das infecções que acometiam os pacientes internados em 2015, 19,3\% eram relacionadas à procedimentos cirúrgicos. ${ }^{3}$ Os eventos infecciosos referentes a estes procedimentos, segundo a Organização Mundial de Saúde (OMS), seriam preveníveis em até $60 \%$ com a aplicação de medidas de prevenção. ${ }^{1}$

Embora avanços tenham sido feitos nas práticas de controle de infecção em relação à estrutura para atendimento, métodos de esterilização e técnica cirúrgica, as ISC continuam sendo uma causa relevante de morbidade, hospitalização prolongada e morte. ${ }^{1}$ A ISC está 
associada a uma taxa de mortalidade que pode variar de $3 \%$ a $75 \%{ }^{4} \mathrm{~A}$ ISC é dentre as IRAS a mais onerosa, com um custo anual estimado de U\$3,3 bilhões, e está associado a quase 1 milhão de dias de internação adicionais anualmente. ${ }^{5-6}$ Em 2014, nos Estados Unidos, foram realizados 14,2 milhões de cirurgias e em média, os custos hospitalares para internações com procedimentos cirúrgicos são mais do que o dobro dos custos para internações sem procedimentos cirúrgicos e as cirurgias de coluna estão entre os procedimentos de maior custo. ${ }^{7}$

Fatores de risco estão associados às ISC em cirurgia de coluna. Dessa forma, a prevenção dessas infecções é complexa e requer a integração de uma série de medidas preventivas antes, durante e após a cirurgia. Os elementos de risco que se destacam são: idade, tabagismo, falta de controle glicêmico no pré, trans e pós operatório, estado nutricional inadequado, diabetes mellitus, doenças prévias não controladas, infecções em outros sítios, tempo de permanência no ambiente de saúde no pré e pós operatório, preparo da pele indevido, quebra de técnicas assépticas, inadequada higiene das mãos, iatrogenias no ato operatório, número de procedimentos invasivos, níveis da cirurgia, próteses e órteses e fatores relacionados ao ambiente. ${ }^{8}$ Outras medidas aplicadas aos procedimentos cirúrgicos também podem influenciar nas taxas de infecção. Dentre elas: o uso de antibiótico profilático fora dos padrões do protocolo (30 a 60 min antes da incisão) e a manutenção do antibiótico após 24 horas da cirurgia, a falta de controle de temperatura corporal durante a cirurgia, e quebras de técnica cirúrgica. Também, o tempo estendido do procedimento e fragilidades na esterilização de materiais, desinfecção de mobiliários e ambiente. ${ }^{9-10}$

Embora a prevenção e o controle de infecção sejam ações desenvolvidas por uma equipe multiprofissional, a enfermagem tem posição de destaque devido a participação em grande maioria dos processos de trabalho relacionados à prevenção, e por isso, é considerado um serviço estratégico para reduzir índices de infecção. ${ }^{11}$ As ações de enfermagem no controle e prevenção das infecções, pautadas em conhecimento e atualização científica, definem atribuição de responsabilidades no cuidado para alcançar níveis de qualidade de desenvolvimento da assistência. 
Adesão aos protocolos de atendimento para a não infecção de sítio cirúrgico de coluna $\mid 4$

A equipe da assistência é a responsável por essas medidas e diante disso, é necessário identificar as fragilidades e fatores de risco relacionados para promover a educação em saúde a fim de ampliar as ações de conscientização e evitar eventos adversos. Ainda, a avaliação dos processos de trabalho é importante para retroalimentar as práticas educativas e fortalecer os protocolos de prevenção de infecção de acordo com o perfil epidemiológico. ${ }^{12}$

A OMS e Agência Nacional de Vigilância Sanitária (ANVISA) Brasil, tem realizado campanhas de conscientização e estabelecido manuais de orientação à estas medidas. Nesse sentido, esses protocolos devem ser aplicados e acompanhados em todos os serviços de saúde. Uma das recomendações para auxiliar nesse processo de cuidado e avaliar a assistência cirúrgica é a implementação de uma Lista de Verificação de Segurança Cirúrgica (LVSC) e checklist relacionado ao atendimento de saúde, os quais preconizam a prevenção de infecção de sítio cirúrgico, melhoria do trabalho em equipe e comunicação, e mensuração do cuidado por meio de indicadores de processos e resultados da assistência cirúrgica. ${ }^{1-2}$

Diante desse contexto questiona-se: as medidas de prevenção de infecção de sítio cirúrgico estão sendo desenvolvidas na prática clínica? Por conseguinte, o objetivo deste estudo foi avaliar a adesão aos protocolos de atendimento para a não infecção de sítio cirúrgico (NISC) de coluna e os fatores associados.

\section{Método}

Estudo transversal, realizado por meio da revisão de 60 prontuários de pacientes submetidos à cirurgia de coluna de 2015 a 2019 e seu desfecho de não infecção (Figura 1) em um hospital de ensino em Santa Cruz do Sul, Rio Grande do Sul, Brasil. Esta pesquisa foi executada por membros integrante da Comissão de Controle de Infecção, utilizando dados de prontuário eletrônico e físico para preenchimento de instrumento específico (ficha de coleta de dados) com variáveis padronizadas. Foram incluídos pacientes submetidos a cirurgia de artrodese, fratura, 
5 | Krummenauer EC, Renner JDP, Menezes RM, Lima TTF, Carneiro M

luxação, hérnia discal e laminectomia desempenhados por apenas uma única equipe cirúrgica. A coleta dos dados e avaliação dos mesmos ocorreram no primeiro semestre do ano de 2020. Todos os pacientes foram avaliados durante a internação e após 90 dias da alta. Foram considerados como critérios de exclusão os pacientes com infecção cirúrgica prévia. Os mesmos foram monitorados durante a internação e até 90 dias após a alta pelo serviço de controle de infecção.

Trata-se de um estudo em que os pacientes submetidos a cirurgias de coluna vertebral foram avaliados em relação ao desfecho de não infecção cirúrgica e à adesão aos protocolos de atendimento. No decorrer dos anos da pesquisa, os protocolos de prevenção de ISC foram revisados e implementados na instituição. A implantação dos cuidados com o banho préoperatório com clorexidina na noite anterior ao procedimento e antibioticoprofilaxia cirúrgica, infundida na indução anestésica de 30 até 60 minutos antes da incisão; e a dose adicional se o procedimento se estender por mais de 4 horas, com tempo de uso de 24 horas, aconteceu no ano de 2012. Já a normotermia, manutenção da temperatura (T) maior ou igual a 35,5든 no pós-operatório imediato e a glicemia menor que $200 \mathrm{mg} / \mathrm{dl}$, mensurado às 6 horas do primeiro dia pós-operatório, foram estabelecidas no ano de 2016, com revisão em 2017. Em 2018, com a implantação da LVSC na instituição, foi estabelecido a checagem da profilaxia antimicrobiana e em 2019, o controle da glicemia.

As variáveis preditoras associadas a NISC, de acordo com a literatura ${ }^{1-2,8,10,13}$ e coletadas para a análise foram: idade, sexo, internação pré-cirúrgica, internação pré-cirúrgica em unidade de terapia intensiva (UTI), controle glicêmico antes da cirurgia, controle glicêmico após a cirurgia, realização de procedimento anterior à cirurgia, banho pré-operatório, banho pré-operatório com clorexidina, higiene oral com clorexidina, degermação da pele com clorexidina, antissepsia da pele com solução combinada de gluconato de clorexidina a $2 \%$ e álcool isopropílico a $70 \%$, antissepsia da pele com clorexidina e iodopovidona, profilaxia antimicrobiana (30 a 60 min antes da incisão), profilaxia estendida ( $>24$ horas), normotermia $\left(35,5\right.$ a $\left.38,3^{\circ} \mathrm{C}\right)$, drenos até 24 horas no pós operatório, internação pós operatória em UTI, internação pós operatória em UTI até 3 dias, 
Adesão aos protocolos de atendimento para a não infecção de sítio cirúrgico de coluna | 6

internação total até 10 dias, internação total de 11-20 dias, internação total de 21-30 dias, internação total maior que 30 dias.

Alguns fatores foram combinados a fim de avaliar o desfecho de não infecção: tempo de internação (internação pré-cirúrgica menor ou igual à 24 horas, não internação pré-cirúrgica em UTI, internação pós cirúrgica em UTI em até 3 dias, internação total de até 10 dias); higiene do paciente pré-operatória (banho pré-operatório com clorexidina e higiene oral com clorexidina); controle de glicemia, profilaxia e normotermia (controle glicêmico antes e após a cirurgia; profilaxia de 30 a 60 min antes da incisão; normotermia, T corporal entre 35,5 e $\left.38,3^{\circ} \mathrm{C}\right)$.

$\mathrm{Na}$ análise das variáveis, foram consideradas como adequadas as que se encaixam como adesão aos protocolos de prevenção de infecção cirúrgica (descritas como “sim”) e como inadequadas as que não atendem a este requisito, bem como, a ausência de registros nos prontuários em relação ao elemento analisado (descritas como "não”).

Para classificação das ISC do sistema nervoso central, utilizou-se os critérios estabelecidos pela ANVISA. ${ }^{2}$ Os dados coletados foram compilados em um banco de dados no programa Excel e analisados estatisticamente por meio do programa Statistical Package for the Social Sciences (SPSS IBM, Armonk, EUA), versão 23.0. Analisou-se frequências e distribuições proporcionais de casos relatados. Avaliou-se variáveis como proporções e variáveis contínuas normalmente distribuídas como médias com desvios-padrão. Usados testes paramétricos para dados com distribuições normais e testes não paramétricos para dados sem distribuições normais. Comparou-se as médias usando testes t de Student e proporções com testes do qui-quadrado.

Medidas de efeito foram utilizadas como diferença de médias ou diferença de proporções, com seus respectivos intervalos de confiança de 95\% (IC 95\%). Para determinar os fatores significativos para NISC, a relação entre não ISC e fatores de risco foi analisada na população deste estudo usando análise univariada. A regressão linear múltipla foi aplicada para as variáveis 
7 | Krummenauer EC, Renner JDP, Menezes RM, Lima TTF, Carneiro M

que apresentaram $\mathrm{p}<0,20$ a fim de considerar fatores de proteção para o desfecho. Um valor de $\mathrm{p}<$ 0,05 foi considerado significante.

O projeto foi submetido, via plataforma Brasil, para apreciação no Comitê de Ética em Pesquisa (CEP), sendo aprovado sob parecer número 3.629 .429 em 08 de outubro de 2019. Além disso, atendendo às Diretrizes e Normas Regulamentadoras de Pesquisas Envolvendo Seres Humanos (Resolução CNS 466/12- 510/2016 - 580/2018, do Ministério da Saúde).



Legenda: ISC: Infecção de Sítio Cirúrgico

Figura 1 - Fluxograma dos pacientes submetidos a cirurgias de coluna, Santa Cruz do Sul, RS, Brasil (2015 a 2019)

\section{Resultados}

Os 60 pacientes foram classificados conforme o desfecho de não infecção de sítio cirúrgico (NISC) e infecção de sítio cirúrgico (ISC) (Figura 1). Os pacientes com NISC tiveram as seguintes características: sexo masculino $32(64 \%)(\mathrm{RR}=0,90 ; p=0,406$; IC 95\% 0,01 - 0,38), com média de idade de 54,94 anos ( \pm 17,49) (IC 95\% 49,06 - 62,07). Os pacientes de NISC foram analisados de 
Adesão aos protocolos de atendimento para a não infecção de sítio cirúrgico de coluna $\mid 8$

acordo com as variáveis associadas para NISC (Tabela 1 e Tabela 2) que foram: higiene oral com clorexidina $(\mathrm{RR}=1,03 ; p=0,023 ; \mathrm{IC} 95 \% 0,00-0,23)$, profilaxia antimicrobiana de 30 a $60 \mathrm{~min}$ antes da cirurgia $\left(\mathrm{RR}=0,97 ; p=0,026\right.$; IC 95\% 0,00 - 0,40), normotermia (T entre $\left.35,5-38,3^{\circ} \mathrm{C}\right)(\mathrm{RR}=0,80$; $p=0,050 ;$ IC 95\% 0,00 - 0,46), internação pós cirúrgica em UTI (até 3 dias) $(\mathrm{RR}=2,00 ; p=0,040 ;$ IC 95\% 0,06 - 0,84), internação maior que 30 dias $(\mathrm{RR}=0,69 ; p=0,017$; IC 95\% 0,03-0,54). A frequência de NISC foi de $83,3 \%(50 / 60) p=0,728 ;$ IC $95 \% 0,00-0,33)$.

$\mathrm{Na}$ Tabelas a seguir, serão abordadas variáveis associadas para NISC, descritas como sim os indicadores de adesão ao protocolo.

Tabela 1 - Análise univariada dos fatores associados para NISC pré-cirúrgicos, Santa Cruz do Sul, Rio Grande do Sul, 2020.

\begin{tabular}{|c|c|c|c|c|}
\hline & \multicolumn{2}{|c|}{$\operatorname{Sem~ISC~}^{*}(\mathrm{n}=50)$} & \multirow{2}{*}{ p - Valor ${ }^{*}$} & \multirow{2}{*}{$\mathrm{RR}^{\S}(\mathrm{IC} \|$ 95\%) } \\
\hline & $\operatorname{Sim}^{+}$ & Não & & \\
\hline Internação pré cirúrgica $>$ " 24 horas & $35(70,0)$ & $15(30,0)$ & 0,522 & $1,08(0,87-1,36)$ \\
\hline Internação pré cirúrgica em UTI** & $17(34,0)$ & $33(66,0)$ & 0,338 & $1,12(0,87-1,45)$ \\
\hline Controle glicêmico antes da cirurgia & $22(44,0)$ & $28(56,0)$ & 0,728 & $0,96(0,76-1,21)$ \\
\hline Procedimento anterior à cirurgia & $6(12,0)$ & $44(88,0)$ & 0,497 & $0,89(0,58-1,34)$ \\
\hline Banho pré operatório & $33(66,0)$ & $17(34,0)$ & 0,717 & $1,04(0,82-1,34)$ \\
\hline Banho água/sabão/clorexidina & $14(28,0)$ & $36(72,0)$ & 0,067 & $1,07(0,85-1,35)$ \\
\hline Higiene oral com clorexidina & $6(12,0)$ & $44(88,0)$ & 0,023 & $1,03(0,74-1,43)$ \\
\hline Degermação clorexidina & $37(74,0)$ & $13(26,0)$ & 0,275 & $0,87(0,71-1,06)$ \\
\hline $\begin{array}{l}\text { Antissepsia clorexidina e álcool } \\
\text { isopropílico }\end{array}$ & $10(20,0)$ & $40(80,0)$ & 1,000 & $1,00(0,78-1,33)$ \\
\hline Antissepsia clorexidina & $27(54,0)$ & $23(46,0)$ & 0,351 & $0,90(0,72-1,12)$ \\
\hline Antissepsia clorexidina e PVPI & $13(26,0)$ & $37(74,0)$ & 0,068 & $1,27(1,11-1,47)$ \\
\hline $\begin{array}{l}\text { Profilaxia antimicrobiana ( } 30 \text { a } 60 \mathrm{~min} \\
\text { antes da incisão) }\end{array}$ & $8(19,5)$ & $33(80,5)$ & 0,026 & $0,97(0,69-1,36)$ \\
\hline
\end{tabular}

ISC: Infecção de Sítio Cirúrgico. ${ }^{\dagger} \mathrm{n}(\%) .{ }^{*}$ Teste do qui-quadrado. ${ }^{\S} \mathrm{RR}$ : Risco Relativo. "IC: Intervalo de Confiança. " >: maior ${ }^{* *}$ UTI: Unidade de Tratamento Intensivo.

Tabela 2 - Análise univariada dos fatores associados para NISC trans e pósoperatórios, Santa Cruz do Sul, Rio Grande do Sul, 2020.

\begin{tabular}{|c|c|c|c|c|}
\hline & \multicolumn{2}{|c|}{$\operatorname{Sem~ISC~}^{*}(n=50)$} & \multirow{2}{*}{$\mathrm{p}$ - Valor ${ }^{*}$} & \multirow{2}{*}{$\mathrm{RR}^{\S}(\mathrm{IC} \|$ 95\%) } \\
\hline & $\operatorname{Sim}^{+}$ & Não ${ }^{\dagger}$ & & \\
\hline $\begin{array}{c}\text { Controle glicêmico após a } \\
\text { cirurgia }\end{array}$ & $27(54,0)$ & $23(46,0)$ & 0,817 & $1,03(0,84-1,25)$ \\
\hline $\begin{array}{c}\text { Profilaxia antimicrobiana } \\
\text { estendida } \leq \pi 24 \text { horas }\end{array}$ & $4(8,0)$ & $46(92,0)$ & 0,120 & $1,22(1,08-1,38)$ \\
\hline Normotermia (temperatura & $27(54,0)$ & $23(46,0)$ & 0,050 & $0,80(0,63-1,00)$ \\
\hline
\end{tabular}


9 | Krummenauer EC, Renner JDP, Menezes RM, Lima TTF, Carneiro M

\begin{tabular}{|c|c|c|c|c|}
\hline \multicolumn{5}{|l|}{ corporal entre 35,5 e $38,3^{\circ} \mathrm{C}^{* *}$ ) } \\
\hline $\begin{array}{c}\text { Dreno até } 24 \text { horas após a } \\
\text { cirurgia }\end{array}$ & $47(94,0)$ & $3(6,0)$ & 0,103 & $0,89(0,68-0,93)$ \\
\hline $\begin{array}{c}\text { Internação pós cirúrgica em } \\
\text { UTI }^{\Uparrow}\end{array}$ & $15(30,0)$ & $35(70,0)$ & 0,069 & $0,80(0,59-1,06)$ \\
\hline $\begin{array}{c}\text { Internação pós cirúrgica em } \\
\text { UTI }^{\natural} \text { até } 3 \text { dias }\end{array}$ & $12(80,0)$ & $3(20,0)$ & 0,040 & $2,00(0,83-4,83)$ \\
\hline Internação total até 10 dias & $14(28,0)$ & $36(72,0)$ & 0,230 & $1,17(0,96-1,42)$ \\
\hline $\begin{array}{c}\text { Internação total de } 11-20 \\
\text { dias }\end{array}$ & $18(36,0)$ & $32(64,0)$ & 0,327 & $1,12(0,91-1,39)$ \\
\hline $\begin{array}{c}\text { Internação total de } 21-30 \\
\text { dias }\end{array}$ & $11(22,0)$ & $39(78,0)$ & 0,889 & $1,12(0,78-1,33)$ \\
\hline Internação total $>^{* *} 30$ dias & $8(16,0)$ & $42(84,0)$ & 0,017 & $0,69(0,44-1,07)$ \\
\hline
\end{tabular}

ISC: Infecção de Sítio Cirúrgico. ${ }^{\dagger} \mathrm{n}(\%)$. "Teste do qui-quadrado. ${ }^{\S}$ RR: Risco Relativo. "IC: Intervalo de

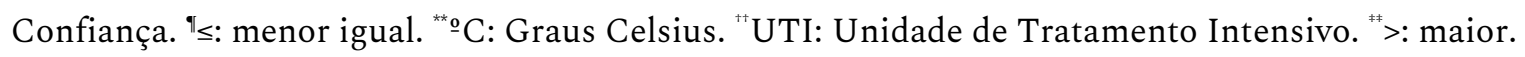

$\mathrm{Na}$ Tabela 3, foram associados alguns elementos aleatórios em relação à característica da internação, higiene pré-operatória do paciente e controle de glicemia, normotermia e profilaxia. Ambas as associações não demonstraram diferença significativa como fator de proteção para NISC.

Dos pacientes com NISC, a média de internação pré-operatória foi 9,56 dias $( \pm 10,20), 15$ (30\%) pacientes foram transferidos para UTI, no pós-operatório, com uma média de 2,6 $\pm 2,03)$ dias de internação e a média de internação hospitalar foi de 19,58 $( \pm 14,17)$ dias.

Das 22 variáveis analisadas, sete foram encontradas fragilidades em relação aos registros em prontuário à adesão aos protocolos de medidas de prevenção: banho pré-operatório 17/50 (34\%) e banho pré-operatório com clorexidina $20 / 50(40 \%)$ e normotermia corporal entre 35,5 a $38,3^{\circ} \mathrm{C}$ 23/50 (46\%). A taxa geral de adesão aos protocolos para NISC foi de $37,4 \%$.

Tabela 3 - Análise univariada de fatores associados para NISC, Santa Cruz do Sul, Rio Grande do Sul, 2020.

\begin{tabular}{|c|c|c|c|c|}
\hline & \multicolumn{2}{|c|}{$\operatorname{Sem~ISC}^{*}(n=50)$} & \multirow{2}{*}{ p - Valor } & \multirow{2}{*}{$\mathbf{R R}^{\S}(\mathrm{IC} \|$ 95\%) } \\
\hline & $\operatorname{Sim}^{+}$ & Não ${ }^{+}$ & & \\
\hline \multicolumn{5}{|l|}{ Internação pré cirúrgica $\leq \mathbb{\rrbracket} 24$ horas; não } \\
\hline internação pré cirúrgica em UTI**; internação & $12(24,0)$ & $38(76,0)$ & $0,327^{* *}$ & 1,14 \\
\hline pós cirúrgica em UTI** até 3 dias; tempo de & & & $(0,00-0,29)$ & $(0,92-1,40)$ \\
\hline \multicolumn{5}{|l|}{ internação total de até 10 dias } \\
\hline Banho pré operatório com clorexidina; higiene & $6(12,0)$ & $44(88,0)$ & $0,857^{\star *}$ & 1,03 \\
\hline
\end{tabular}


Adesão aos protocolos de atendimento para a não infecção de sítio cirúrgico de coluna | 10

oral com clorexidina

Controle glicêmico antes e após à cirurgia;

profilaxia de 30 a 60 min antes da incisão;

normotermia (Temperatura corporal entre

35,5 e $\left.38,3^{\circ} \mathrm{C}^{+1}\right)$

$$
(0,01-0,28) \quad(0,74-1,43)
$$

$$
\begin{aligned}
& 7(14,0) \quad 43(86,0) \quad 0,734^{\star *} \quad 1,05 \\
& (0,00-0,42) \quad(0,53-1,33)
\end{aligned}
$$

ISC: Infecção de Sítio Cirúrgico. ${ }^{+} \mathrm{n}(\%)$. ${ }^{*}$ Teste do qui-quadrado. ${ }^{\S} \mathrm{RR}$ : Risco Relativo. "IC: Intervalo de Confiança. " $\leq$ : menor igual. ${ }^{* *}$ UTI: Unidade de Tratamento Intensivo. ${ }^{+t}$ o $\mathrm{C}$ : Graus Celsius ${ }^{* *}>$ : maior.

A normotermia foi a única variável que manteve associação como fator associado a NISC (RR, 0,16; IC de 95\%, -0,920 - -0,015) (Tabela 4). As demais variáveis apresentaram no modelo de regressão $p>0,20$.

\begin{tabular}{|c|c|c|c|}
\hline & $\begin{array}{c}\text { Sem ISC }^{*} \\
(\mathrm{n}=50)\end{array}$ & $p$ - Valor & $\mathrm{RR}^{\S}(\mathrm{IC} \|$ 95\%) \\
\hline $\begin{array}{c}\text { Normotermia (Temperatura corporal } \\
\left.\text { entre } 35,5 \text { e } 38,3^{\circ} \mathrm{C}\right)\end{array}$ & $27(54)^{+}$ & 0,038 & $0,16(-0,92--0,01)$ \\
\hline $\begin{array}{c}\text { Internação pós cirúrgica em UTI" até } 3 \\
\text { dias }\end{array}$ & $12(80)^{\dagger}$ & 0,148 & $0,10(-0,36--0,87)$ \\
\hline
\end{tabular}

Tabela 4 - Regressão linear múltipla de variáveis preditoras para NISC, Santa Cruz do Sul, Rio Grande do Sul, 2020.

ISC: Infecção de Sítio Cirúrgico. ${ }^{\dagger} \mathrm{n}(\%) .{ }^{*}$ Teste do qui-quadrado. ${ }^{\S}$ RR: Risco Relativo. "IC: Intervalo de Confiança. " UTI: Unidade de Tratamento Intensivo.

\section{Discussão}

As ISC são complicações frequentes e uma das principais causas de morbidade pósoperatória (internação hospitalar prolongada e uso de antimicrobianos) e mortalidade, além de custos diretos e indiretos. ${ }^{14}$ Apesar da implementação de medidas preventivas que controlam os fatores de proteção relacionados ao paciente, bem como os fatores independentes (como tempo de internação pré e pós operatória, controle glicêmico, de temperatura corporal e preparo da pele, antibioticoprofilaxia e tempo de uso de drenos) a infecção em cirurgia da coluna é relevante. ${ }^{15-16}$ Para controlar estes fatores se faz necessário a aplicação de medidas a fim de reduzir estes riscos. Em um estudo de cirurgias de coluna foi observado um aumento de proteção de $82 \%$ para NISC após a administração de pacotes de cuidados. ${ }^{17}$ Nesta pesquisa, na análise dos fatores associados, 
não se constatou maior segurança para NISC, pois se tornou evidente a fragilidade da aplicação do protocolo de medidas de prevenção de infecção relacionadas ao sítio cirúrgico nos participantes do estudo. Esta classificação de inadequado dificulta a análise associada, pois todos os elementos analisados precisam estar apropriados.

Nesta análise os fatores para NISC foram higiene oral com clorexidina, antissepsia com clorexidina e iodopovidona, profilaxia antimicrobiana de 30 a 60 min antes da incisão, normotermia, menor tempo de internação pós operatório em UTI, no entanto, na regressão linear permaneceu apenas a normotermia como variável independente. A normotermia é uma

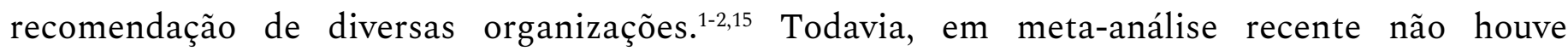
associação com ISC, ${ }^{18}$ colocando em dúvida novamente tais conceitos. $\mathrm{Na}$ análise das demais variáveis que compõem o pacote de medidas não se evidencio relação ao fator de proteção para NISC, o que também foi encontrado em outro estudo. ${ }^{19}$

A higiene oral perioperatória com uso de clorexidina reduz as infecções pós-operatórias do trato respiratório inferior e do sítio cirúrgico. A descontaminação no pós-operatório é uma estratégia que também é relevante. É uma intervenção fácil e pode ser realizada pelos próprios pacientes, principalmente em cirurgias de alta complexidade..$^{18,20}$

A solução de preparação da pele é um fator importante na prevenção de ISC e os compostos à base de iodo podem ser semelhantes à clorexidina. Existem controvérsias em estudos e vários produtos com apresentações e concentrações diferentes para este fim com desfechos similares. ${ }^{21-23}$

Estudo salienta que os pacientes que não receberam profilaxia antibiótica tiveram 2,57 vezes mais chance de desenvolver ISC em comparação com aqueles que receberam profilaxia antibiótica. ${ }^{24} \mathrm{O}$ tempo adequado da administração é crítico para a eficácia da profilaxia, e a forma prolongada pode ser prejudicial, pois promove bactérias resistentes e aumenta a incidência de complicações e custos agregados. A resistência a essa classe de medicamentos é uma ameaça à saúde pública global e tem como uma das principais causas o uso excessivo de antibióticos. ${ }^{25}$ 
Adesão aos protocolos de atendimento para a não infecção de sítio cirúrgico de coluna | 12

Este estudo não conseguiu demonstrar estatisticamente como fator de proteção independente para NISC o tempo de permanência hospitalar em UTI pré e pós operatório. Uma investigação concluiu que não houve associação significativa entre tempo de internação préoperatório em UTI e risco de ISC mas confirmou que o tempo de permanência pós-operatório foi significativamente associado. ${ }^{26}$ Já outra pesquisa em pacientes submetidos à cirurgia cardíaca a permanência hospitalar pré-operatória prolongada foi significativamente associada com o desenvolvimento de ISC com uma OR ajustada por dia de 1,38 (IC 95\%: 1,02-1,86; p = 0,036). ${ }^{27}$ Esses achados precisam ser considerados no manejo clínico do paciente, a fim de otimizar o tempo de permanência do paciente no ambiente de saúde e evitar o uso desnecessário de antibióticos.

O controle glicêmico pós-operatório estava adequado em aproximadamente metade dos pacientes analisados. Sugere-se o monitoramento rigoroso pela equipe cirúrgica, especialmente naqueles com alto risco de ISC e ressalta-se que este indicador é de fácil mensuração e deve ser priorizado. Os resultados de um estudo mostram que o controle glicêmico rígido imediatamente após a cirurgia reduz significativamente as taxas de infecção pós-operatória pois estes pacientes apresentam uma taxa de hipoglicemia maior no pós-operatório. ${ }^{28}$

Os pacientes que apresentaram NISC tiveram uma permanência hospitalar diminuída o que corrobora para avaliar a eficácia do atendimento e a qualidade e a segurança da assistência do paciente cirúrgico. O monitoramento e o controle dos fatores de proteção para NISC fazem parte do pacote de medidas de prevenção da ISC permitindo o direcionamento das ações dos profissionais na adoção de práticas que reduzam a incidência de infecções e complicações inerentes à mesma. A enfermagem, como integrante de uma equipe multidisciplinar, pode desenvolver ações baseadas nestes protocolos e para isso é necessário a ampliação e o fortalecimento de políticas de educação permanente a fim de reconhecer precocemente os elementos de risco e estabelecer estratégias para minimizar complicações, garantir a segurança e a qualidade do atendimento prestado ao paciente. ${ }^{9}$ 
Para isso, é necessário certificar um conjunto de ações que visam aumentar a segurança do paciente cirúrgico por meio de um incentivo à adesão às boas práticas em sua completude. A fragilidade dos indicadores de concordância dos protocolos neste estudo será importante para redirecionar a prática da equipe em relação à necessidade do aprimoramento da segurança do paciente cirúrgico e prevenção de infecção.

Uma alternativa recomendada para auxiliar na incorporação das medidas de cuidados e ampliar os indicadores de adesão aos protocolos de medidas de prevenção de infecção é a implementação de checklist de verificação e conscientização das equipes por meio de processos educativos. Para aplicação destes instrumentos, fazem-se necessários, além da sensibilização, mudança na percepção da equipe cirúrgica e ajuste dos fluxos de trabalho, mas é essencial para a padronização de cuidados na lista de checagem, além de fortalecer a comunicação entre a equipe.

Uma revisão sistemática estudou os problemas da aplicação da LVSC e destaca que as dificuldades de implementação são multifatoriais com relevante interferência da complexidade clínica. O principal destaque é em relação ao conflito qualidade versus produtividade em relação aos fluxos de trabalho. É importante estabelecer um clima de segurança por meio da interação das políticas e protocolos de atendimento fortalecidos pelo trabalho em equipe e comunicação dos profissionais. ${ }^{29}$ Estas evidencias também foram destacadas em outro estudo que sinalizou as fragilidades de um instrumento de verificação que apresenta vulnerabilidades de preenchimento e salienta a importância do apoio da administração e chefias para o fortalecimento desta barreira adicional. ${ }^{30}$

Embora muitas variáveis analisadas não integram a lista de verificação, estas ações estão recomendadas e validadas em protocolos institucionais, adequados à realidade do serviço. O registro em prontuários e em instrumentos específicos para prevenção de infecção é de competência da equipe de atendimento e precisa ser fortalecido por meio do desenvolvimento de habilidades, pois é uma forma de qualificar a comunicação e assegurar a execução na prática diária, bem como, fornecer subsídios para uma atuação da equipe de forma pró ativa. Por meio deste, levanta indicadores para 
Adesão aos protocolos de atendimento para a não infecção de sítio cirúrgico de coluna | 14

retroalimentar os processos educacionais da equipe assistencial, indispensáveis no controle de infecção, e fortalece a cultura de segurança através da redução de eventos adversos relacionados.

Este estudo teve como limitadores a análise com poucos participantes, unicêntrico, que analisou alguns fatores de atendimento envolvendo pré, trans e pós operatório, não incluindo estado físico do paciente e seus fatores de risco, habilidades da equipe cirúrgica, comportamento dos profissionais da assistência trans operatória, práticas de esterilização dos materiais cirúrgicos e demais procedimentos invasivos. Variáveis não foram coletadas de alguns pacientes por não estarem registradas no prontuário, interferindo na avaliação dos processos de trabalho e sinalizando a necessidade de revisão do modelo de gestão e fortalecimento das ações de cuidados para prevenir infecções. Esses dados podem ser úteis para redirecionar a prática, fortalecer a LVSC e o check list de medidas de prevenção, além de ofertar qualidade na assistência e segurança ao paciente cirúrgico.

\section{Conclusão}

Os estudos acerca deste tema destacam que as infecções têm como causa uma variedade de fatores, e o controle de cada um deles é importante, embora nesta pesquisa, encontrou-se apenas a normotermia como fator de proteção para NISC e insinua-se que este desfecho poderia ser mais abrangente com uma maior conformidade em relação aos protocolos. Ressalta-se que a adesão aos processos de atendimento ao paciente cirúrgico é primordial para a proteção das infecções e eventos adversos, e a educação permanente das equipes é imprescindível para o ajuste destas medidas, garantindo a qualidade da assistência prestada.

Muitas destas ações são controladas e ou executadas pela enfermagem que acompanha o paciente no peri, trans e pós-operatório por isso tem a responsabilidade de reduzir as complicações relacionadas. Identificaram-se falta de registros e não execução de procedimentos ou checagem relacionados aos fatores de risco. Estas ações precisam estar pautadas em evidências científicas e validadas para garantir a segurança do paciente. Para que estas práticas façam parte de um 
atendimento seguro, é necessário conhecimento técnico a respeito do assunto, o que requer revisão dos temas frequentemente e incentivo à adesão a estes protocolos.

Destaca-se que o pacote de medidas de prevenção de infecção é um conjunto de critérios para se obter melhores resultados de atendimento e que a maior probabilidade de proteção se dá com a adesão a estas normas em sua maior proporção, do que quando aplicadas isoladamente. Salienta-se a importância da participação da equipe de enfermagem no processo de prevenção das ISC, já que ela presta assistência por maior tempo ao paciente, o que perfaz maior volume e criticidade de cuidados. Espera-se que a fragilidade dos indicadores de adesão aos protocolos encontrados neste estudo possa servir de embasamento para redirecionar a prática. O enfermeiro precisa avaliar os fatores predisponentes e de riscos à infecção, de acordo com os protocolos, e adotar medidas preventivas e educacionais para todos os sujeitos envolvidos, por meio de um processo de sensibilização coletiva, que prevê a execução de ações previstas e registros da checagem das mesmas nos prontuários de atendimento. Esta dinâmica, além de fortalecer a prática clínica da equipe de enfermagem, demonstra o envolvimento em ações de segurança para diminuir a ocorrência de complicações pós-cirúrgica. Sugere-se o fortalecimento da LVSC e check list de medidas de prevenção de infecção que podem ofertar benefícios para os profissionais por meio do fortalecimento da comunicação e para o paciente, qualidade na assistência e promoção da segurança.

Evidencia-se que a avaliação dos processos de trabalho foi dificultada pela falta de registros e interferiu na qualidade e segurança da assistência ao paciente cirúrgico, sinalizando a necessidade da revisão do modelo de gestão. Esses dados podem ser úteis para o planejamento cirúrgico, aconselhamento do paciente e esforços para melhorar a segurança. Sugere-se que estudos prospectivos e controlados, multicêntricos, sejam realizados para comprovar resultados positivos.

\section{Referências}

1. World Health Organization (WHO). Preventing surgical site infections: implementation approaches for evidence-based recommendations [Internet]. Geneva (CH): World Health Organization; 2018 [cited 2020 Jun 
Adesão aos protocolos de atendimento para a não infecção de sítio cirúrgico de coluna | 16

22]. Available from: https://apps.who.int/iris/handle/10665/273154

2. Agência Nacional de Vigilância Sanitária (ANVISA). Nota Técnica GVIMS-GGTES N. 03/2019 - Critérios diagnósticos das infecções relacionadas à assistência à saúde [Internet]. Brasília (DF): ANVISA; 2019 [acesso em 2020 out 10]. Disponível em: https:/www.gov.br/anvisa/pt-br/centraisdeconteudo/publicacoes/servicosdesaude/notastecnicas/nota-tecnica-no-03-2019-gvims-ggtes-anvisa.pdf/view

3. Magill SS, O'Leary E, Janelle SJ, Thompson DL, Dumyati G, Nadle J, et al. Changes in prevalence of health careassociated infections in U.S. hospitals. N Engl J Med. 2018;1:379(18):1732-44. doi: 10.1056/NEJMoa1801550

4. Awad SS. Adherence to surgical care improvement project measures and post-operative surgical site infections. Surg Infect (Larchmt). 2012;13(4):234-7. doi: 10.1089/sur.2012.131

5. Zimlichman E, Henderson D, Tamir O, Franz C, Song P, Yamin CK, et al. Health care-associated infections: a meta-analysis of costs and financial impact on the US health care system. JAMA Intern Med. 2013;173(22):2039-46. doi: 10.1001/jamainternmed.2013.9763

6. Lissovoy G, Fraeman K, Hutchins V, Murphy D, Song D, Vaughn BB. Surgical site infection: incidence and impact on hospital utilization and treatment costs. Am J Infect Control. 2009;37(5):387-97. doi: 10.1016/j.ajic.2008.12.010

7. Yao R, Zhou H, Choma TJ, Kwon BK, Street J. Surgical site infection in spine surgery: who is at risk? Global Spine J. 2018;8(4):5S-30S. doi: 10.1177/2192568218799056

8. Zhou J, Wang R, Huo X, Xiong W, Kang L, Xue Y. Incidence of surgical site infection after spine surgery: a systematic review and meta-analysis. Spine (Phila Pa 1976). 2020;45(3):208-16. doi: 10.1097/BRS.0000000000003218

9. Carvalho RLR, Campos CC, Franco LMC, Rocha AM, Ercole FF. Incidence and risk factors for surgical site infection in general surgeries. Rev Latinoam Enferm. 2017;25:e2848. doi: 10.1590/1518-8345.1502.2848

10. Mok WQ, Ullal MJ, Su S, Yiap PL, Yu LH, Lim SMM, et al. An integrative care bundle to prevent surgical site infections among surgical hip patients: a retrospective cohort study. Am J Infect Control. 2019;47(5):540-4. doi: 10.1016/j.ajic.2018.10.011

11. Souza KV, Serrano SQ. Saberes dos enfermeiros sobre prevenção de infecção do sítio cirúrgico. Rev SOBECC. 2020;25(1):11-6. doi: 10.5327/Z1414-4425202000010003

12. Cardoso MLM, Costa PP, Costa DM, Xavier C, Souza RMP. A Política Nacional de Educação Permanente em Saúde nas escolas de saúde pública: reflexões a partir da prática. Ciênc Saúde Colet. 2017;22(5):1489-500. doi: 10.1590/1413-81232017225.33222016

13. National Institute for Health and Care Excellence (NICE). Prevention and control of healthcareassociated infections [Internet]. London: NICE; 2021 [cited 2020 Jun 22]. Available from: https://pathways.nice.org.uk/pathways/prevention-and-control-of-healthcare-associated-infections

14. Gandhi SV, Schulder M. Spinal infections [Internet]. Rollin Meadows (USA): American Association of Neurological Surgeons; 2020 [cited 2020 Jun 22]. Available from: https://www.aans.org/en/Patients/Neurosurgical-Conditions-and-Treatments/Spinal-Infections

15. National Healthcare Safety Network National Healthcare Safety Network (NHSN). Patient safety component manual [Internet]. 2020 [cited 2020 Jun 22]; Available from: 
https://www.cdc.gov/nhsn/PDFs/pscManual/pcsManual_current.pdf

16. Nagashima H, Tanishima S, Tanida A. Diagnosis and management of spinal infections. J Orthop Sci. 2018;23(1):8-13. doi: 10.1016/j.jos.2017.09.016

17. Yamada K, Abe H, Higashikawa A, Tonosu J, Kuniya T, Nakajima K, et al. Evidence-based care bundles for preventing surgical site infections in spinal instrumentation surgery. Spine (Phila $\mathrm{Pa}$ 1976). 2018;43(24):1765-73. doi: 10.1097/BRS.0000000000002709

18. Bu N, Zhao E, Gao Y, Zhao S, Bo W, Kong Z, et al. Association between perioperative hypothermia and surgical site infection: a meta-analysis. Medicine (Baltimore). 2019;98(6):e14392. doi: 10.1097/MD.00000000000014392

19. Andrade LS, Siliprandi EMO, Karsburg LL, Berlesi FP, Carvalho OLF, Rosa DS, et al. Surgical site infection prevention bundle in cardiac surgery. Arq Bras Cardiol. 2019;112(6):769-74. doi: 10.5935/abc.20190070

20. Pedersen PU, Larsen P, Håkonsen SJ. The effectiveness of systematic perioperative oral hygiene in reduction of postoperative respiratory tract infections after elective thoracic surgery in adults: a systematic review. JBI Database System Rev Implement Rep. 2016;14(1):140-73. doi: 10.11124/jbisrir-2016-2180

21. Ghobrial GM, Wang MY, Green BA, Levene HB, Manzano G, Vanni S, et al. Preoperative skin antisepsis with chlorhexidine gluconate versus povidone-iodine: a prospective analysis of 6959 consecutive spinal surgery patients. J Neurosurg Spine. 2018;28(2):209-14. doi: 10.3171/2017.5.SPINE17158

22. Chen S, Chen JW, Guo B, Xu CC. Preoperative antisepsis with chlorhexidine versus povidone-iodine for the prevention of surgical site infection: a systematic review and meta-analysis. World J Surg. 2020;44(5):1412-24. doi: 10.1007/s00268-020-05384-7

23. Dörfel D, Maiwald M, Daeschlein G, Müller G, Hudek R, Assadian O, et al. Comparison of the antimicrobial efficacy of povidone-iodine-alcohol versus chlorhexidine-alcohol for surgical skin preparation on the aerobic and anaerobic skin flora of the shoulder region. Antimicrob Resist Infect Control. 2021;10(1):17. doi: 10.1186/s13756-020-00874-8

24. Alamrew K, Tadesse TA, Abiye AA, Shibeshi W. Surgical antimicrobial prophylaxis and incidence of surgical site infections at Ethiopian tertiary-care teaching hospital. Infect Dis (Auckl). 2019;27;12:1178633719892267. doi: 10.1177/1178633719892267

25. Purba AKR, Setiawan D, Bathoorn E, Postma MJ, Dik JWH, Friedrich AW. Prevention of surgical site infections: a systematic review of cost analyses in the use of prophylactic antibiotics. Front Pharmacol. 2018;18;9:776. doi: 10.3389/fphar.2018.00776

26. Mujagic E, Marti WR, Coslovsky M, Soysal SD, Mechera R, von Strauss M, et al. Associations of hospital length of stay with surgical site infections. World J Surg. 2018;42(12):3888-96. doi: 10.1007/s00268-018-4733-4 27. Sulzgruber P, Schnaubelt S, Koller L, Laufer G, Pilz A, Kazem N, et al. An extended duration of the preoperative hospitalization is associated with an increased risk of healthcare-associated infections after cardiac surgery. Sci Rep. 2020;10(1):8006. doi: 10.1038/s41598-020-65019-8

28. Wang YY, Hu SF, Ying HM, Chen L, Li HL, Tian F, et al. Postoperative tight glycemic control significantly reduces postoperative infection rates in patients undergoing surgery: a meta-analysis. BMC 
Adesão aos protocolos de atendimento para a não infecção de sítio cirúrgico de coluna | 18

Endocr Disord. 2018;22;18(1):42. doi: 10.1186/s12902-018-0268-9

29. Bergs J, Lambrechts F, Simons P, Vlayen A, Marneffe W, Hellings J. Barriers and facilitators related to the implementation of surgical safety checklists: a systematic review of the qualitative evidence. BMJ Qual Saf. 2015;24(12):776-86. doi: 10.1136/bmjqs-2015-004021

30. Tostes MFP, Galvão CM. Lista de verificação de segurança cirúrgica: benefícios, facilitadores e barreiras na perspectiva da enfermagem. Rev Gaúcha Enferm. 2019;40(N Esp):e20180180. doi: 10.1590/1983-1447.2019.20180180

Editora Científica: Tânia Solange Bosi de Souza Magnago

Editora Associada: Rhanna Emanuela Fontenele Lima de Carvalho

Fomento / Agradecimento: apoio da Coordenação de Aperfeiçoamento de Pessoal de Nível Superior - Brasil (CAPES) - Código de Financiamento 001.

\section{Autor correspondente}

Eliane Carlosso Krummenauer

E-mail: elianek@unisc.br

Endereço: Thomas Flores 1064, Bloco A2, Apto 302, Centro, Santa Cruz do Sul, RS CEP: 96810078

\section{Contribuições de Autoria}

\section{1 - Eliane Carlosso Krummenauer}

Concepção ou desenho, análise e/ou interpretação dos dados, revisão final com participação crítica e intelectual no manuscrito.

\section{2 - Jane Dagmar Pollo Renner}

Concepção ou desenho, análise e/ou interpretação dos dados, revisão final com participação crítica e intelectual no manuscrito.

\section{3 - Rochele Mosmann Menezes}

Concepção ou desenho, análise e/ou interpretação dos dados, revisão final com participação crítica e intelectual no manuscrito.

\section{4 - Telmo Tiburcio Fortes Lima}

Concepção ou desenho, análise e/ou interpretação dos dados, revisão final com participação crítica e intelectual no manuscrito.

\section{5 - Marcelo Carneiro}

Concepção ou desenho, análise e/ou interpretação dos dados, revisão final com participação crítica e intelectual no manuscrito.

\section{Como citar este artigo}

Krummenauer EC, Renner JDP, Menezes RM, Lima TTF, Carneiro M. Adherence to care protocols for noninfection of the surgical site of the spine. Rev. Enferm. UFSM. 2021 [Accessed in: Year Month Day]; vol.11 e78: 1-18. DOI: https://doi.org/10.5902/2179769264885 